\title{
A Critical Appraisal of the Ban on Serological Tests for Tuberculosis Usefulness of Serological Monitoring of Tuberculosis Antibodies during the Treatment of TB Patients
}

\section{Roland Maes*}

Anda Biologicals, Strasbourg, France

*Corresponding author: Roland Maes, Anda Biologicals, Strasbourg, France, Tel: +0033388501948; Email: anda.mars@wanadoo.fr

Received date: May 22, 2016, Accepted date: June 28, 2016, Published date: June 30, 2016

Copyright: (c) 2016 Maes R. This is an open-access article distributed under the terms of the Creative Commons Attribution License, which permits unrestricted use, distribution, and reproduction in any medium, provided the original author and source are credited.

\section{Abstract}

In 2011, the WHO banned the use of serological tests for the detection of antibodies against mycobacteria. This ban rests on a meta-analysis that observed a disparity in accuracy and specificity of results reported in various studies using this technique. All the included results were either level II observational studies or level III expert opinions. The immense majority of studies on other aspects of TB, as microscopy and vaccine efficacy, also pertain to these levels and present the same spread of results. The WHO policy statement rests on incorrect and invalid evidence. The experts analysed a pool of published studies of widely divergent quality, using different serological markers, and drew thereupon conclusions on quality of the serological test itself instead of on the quality of the studies analysed. In addition, the authors of the meta-analysis report results that are sometimes incorrect, drew conclusions on incomplete data, removed publications from their study using dubious reasons, lumped together results that should have been analysed separately and finally failed to take into consideration the value of the golden diagnostic methods used today. I intend to prove that serology is a very useful complementary tool in the diagnostic and prognostic of mycobacterial infections and that, had the WHO based their analysis on the complete information available, they would have supported the use of some serological tools for the diagnosis of TB instead of banning them.
\end{abstract}

Keywords: Tuberculosis; TB-serology; Level I evidence; TB diagnostic; TB prognostic

\section{Introduction}

The monitoring of the humoral immune status of TB patients is considered useless by the mycobacterial community because, in 1974, Middlebrook reported no benefit from a transfer of immune serum to rabbits experimentally challenged with aerobic TB [1]. The uselessness of humoral antibodies was therewith supposedly demonstrated and comforted by many fruitless attempts to develop a test for the detection of human antibodies against TB. The mycobacterial community did not take into consideration the fact that humans and rabbits are different species which may not react similarly to a TB challenge, nor that the type of antibody used to inactivate the antigen is crucial [2]. The importance of the immunological aspect of TB and leprosy was determined in 1990 [3] but ignored by the scientific community. This importance was again stressed in 1998 [4] but given no heed: the dogma had been established and became immutable. Reduction of the TB problem to detection and compliance without due consideration of the immunological status of each individually treated patient is still the current approach [5]. Contemporary textbooks on clinical tuberculosis largely ignore the humoral immune responses of patients, as if none occurs or else is without any importance. No concern is shown either for the influence of the individual immune response of the patients on the outcome of a treatment or for the influence of the applied drugs on the immune system of the patient. All efforts are directed at the detection of the pathogen, which triggers standardized treatment indistinctly applied to all. New sophisticated methods for antigen detection, search for new drugs and new vaccines demand huge public subventions and lavish expenditures on research and development produce an impressive quantity of results but fail to produce qualitative applications. Investigators who function within this paradigm prefer to question the validity of facts that challenge their paradigm rather than question the paradigm itself. The optimistic belief that all that is needed to eradicate TB is the detection of the pathogen together with the use of vaccine and drugs to "stamp the bug out" have been shattered by its recent upsurge that is in majority linked to diagnostic failure.

The re-emergence of TB despite the standardized diagnostic and treatment methods indicates that there is a problem in the understanding of its pathologic process and that this process is in great need of revision. There is now a large body of evidence that proves that there is a humoral response during tuberculosis and that this humoral response is linked to disease outcome. Serology may thus help to improve the understanding of $\mathrm{TB}$ pathology. The detection of antibodies in association with antigen-detection tests have been found useful for diagnosis and prognosis [6,7] as well as to monitor illness [8]. In particular, the ELISA tests for anti-TB antibodies manufactured by Anda Biologicals [9] have been used in India for over 25 years to diagnose TB cases that cannot be detected by conventional methods. The decision of the WHO to ban the use of antibody detection for TB diagnosis $[10,11]$ is based on a single meta-analysis report [12] that, on close inspection, was poorly made and biased [13]. Furthermore, this analysis fails to take into account the poor results obtained with conventional TB diagnosis methods. This decision by the WHO deprived the clinicians from a diagnostic tool that has proven to be of invaluable assistance in $\mathrm{TB}$ diagnosis and prognosis despite claims to the contrary. 


\section{The WHO ban on TB-serodiagnostics}

The WHO pronounced in 2011 a ban on the serodiagnosis of tuberculous infections $[10,11]$. This decision was based on the results of a meta-analysis of published evaluations of the accuracy and specificity of antibody diagnostic tests [12], and on a cost-effectiveness study performed in India on an hypothetic population [14]. The main criticism advanced by WHO to justify the ban was the spread from $0 \%$ to $100 \%$ (sensitivity, specificity) of some results obtained in different studies and the absence of a stringent evaluation process applied to the tests analysed [15].

These rejected studies were Level II observational studies and Level III expert opinion studies, which is also the case for more than half of the studies used to establish current recommendations of the Infectious Diseases Society of America [16]. A recent generation of doctors wants to make decisions on evidence-based medicine and applies the conclusions of randomized, controlled trials to clinical decision making, i.e. Level I evidence [15]. However, the influence of a randomized controlled trial-no matter how well conducted-pales in comparison with the silent despair of a MDR-TB patient in category II failure. Adverse anecdote Level IV evidence [17] is more convincing than even the tightest of confidence intervals. Anecdotal evidence is an inherent part of medicine. Medicine was, is and will always be an art.

The WHO claims that new diagnostic tests must meet the level of performance reached by microscopy taken as the gold standard, i.e. $75 \%$ sensitivity and $98 \%$ specificity [18]. The claimed performance of microscopy was obtained by evaluating the test in regions with a very high TB incidence and using only adult patients with secondary pulmonary $\mathrm{TB}$. With a high TB incidence, the vast majority of the patients analysed were $M$. tuberculosis microscopy-positive and the specificity was thus near $100 \%$. The lower value reported for the sensitivity $(75 \%)$ was traced to errors in the manipulation and examination of the microscopic slides. A more realistic evaluation of the sensitivity varies from $20 \%$ to $80 \%$ in pulmonary cases, due to lack of electricity, dirty lenses, contamination in stains, poor quality of sputum, fatigue of the microscopist, primary cases and paucibacillary cases. The sensitivity of microscopy in very young children and in the elderly is also very low due to their inability to produce the required sputum sample. In addition, microscopy does not detect extrapulmonary cases, which account for at least $30 \%$ of all TB patients. These last three groups of patients belong to the TB population and their exclusion when determining the sensitivity of a diagnostic test is not justified, yet repeatedly done where microscopy is concerned.

\section{Flaws in the meta-analysis used by the WHO to decide on banning serological TB tests}

For a large number of studies used in the meta-analysis that drove the WHO to ban serological tests for TB, the authors accuse the authors of the clinical trials to have conflicts of interests on the ground that the diagnostic kits used in the studies were donated by the manufacturers. This is a standard practice in all clinical trials, included those performed by the WHO itself, as was the case for their analysis of TB-rapid tests, as well as all the trials performed by FIND while evaluating new diagnostic tests for TB, malaria and other tropical diseases. As a general rule, it is asked that the manufacturer bears at least the cost of the test being evaluated. The real question that should have been asked was: did the manufacturer control the publication of the results. In most small trials of that nature, this is not the case, the independence of the investigators being guaranteed by agreements and contracts prior to the trials.

WHO claims that the accuracy of investigated tests is even lower than reported because bad results would not have been published? This assumption is proven false by the meta-analysis itself that included many "negative" publications. The authors of the meta analysis present an assumption that seems true on the surface but that does not take into account two facts: 1) publications on clinical trials differ from those reporting fundamental science in that negative results are just as important as positive ones and 2) the drive for investigators to publish is stronger than any pressure most diagnostic test manufacturer can exert to prevent it.

The authors claim to have accurately reported the result of the trials they included in their meta-analysis. This is not true on several occasions. For example, results concerning pulmonary cases, obtained in Gevaudan's study [19] were not mentioned in the meta analysis and only extra-pulmonary cases were considered but no difference was drawn between IgG and IgM antibodies for the evaluation of the results, which confused the outcome. Similarly, the results obtained with pulmonary cases by Alifano [20] are included in the metaanalysis, but not those reported in the same paper and a subsequent paper [21] on extrapulmonary cases. The meta-analysis made no difference between IgG, IgM and IgA antibodies, while each has their separate use in diagnosis, as is the case in other diseases such as Mononucleosis and Dengue. IgM detection would be used to detect primary disease in children and adults, the fact that the trials have a low percentage of IgM positive cases only indicates, as with other diseases, that most patients suffer from secondary infections. IgG would be used to confirm secondary or long-standing disease. Detection of IgA has a particular use in TB, as this class of antibodies can still be detected in most but not all cases of immuno-suppression. To lump the results of all these tests together is a gross mistake that indicates that the authors did not understand TB pathogenesis. In addition, the American Statistical Association warned that the reliance on statistical analysis using the $\mathrm{P}$ value to issue policy directives is fundamentally erroneous: "The society's members had become increasingly concerned that the $P$ value was being misapplied in ways that cast doubt on statistics generally. In its statement, the ASA advises researchers to avoid drawing scientific conclusions or making policy decisions based on P values alone" [13].

The WHO policy statement claims that no tests were made on infants and children. I report here 7 authors who published on this subject [22-28]. As indicated above, some of these studies are negative, as they report poor sensitivity in primary cases.

The policy statement of WHO claims that only one test (SDHO rapid test) was made on HIV populations. I report here four studies on the subject [29-32]. All reported poor sensitivity. Yet, the study [32] referenced as \#5 in the indicting meta-analysis, announces $50 \%$ sensitivity for the Anda-TB test in HIV- patients. Production of humoral antibodies in HIV-infected subjects is rare because the immuno-depression inflicted by HIV also affects TB: the negative results observed are not "false negatives" but "true negatives" traced to immuno-depression. The study [32] mentioned in reference \#5 of the meta analysis presents a particular interest because it also focused on the identification of latent infections. Basing its judgment on references \#3 and \#24, it reported for the Anda Biologicals TB ELISA a positivity rate of $30.8 \%$, which was said to be about 10 times higher than the expected conversion rate of $3 \%$ to $5 \%$ from latent TB infection to active TB infection rates. The cited reference \#3 is the guidelines of 
the American Thoracic Society [33]. These guidelines accept the 4.7\% conversion rate that was found in a study on UK school children aged 14-19 years [34]. However, TB conversion can occur more than 10 years after infection and will increase with decreasing health: a conversion rate measured in the 14-19 year old segment of the population cannot be extended to a whole population. In addition, the conversion rate in the UK, a low TB incidence country where the majority of the population is healthy, will be different from that in a high TB incidence country, such as India, where the study on the Anda-TB test was performed. In fact, reference $\# 24$ of the same publication [33] refers to a US study that upgrades this evaluation to at least $20 \%$ [35]. This evaluation of $20 \%$ or more of reactivation risk reflects more accurately the true level of the conversion rate in a general population in regions with a high TB incidence. If the authors of the different studies [12,32] had drawn correct conclusions from their own data and references, the Anda-TB test would have appeared as an excellent test to detect latent infections and the WHO would not have banned it.

We acknowledge that tests based on different immunological responses cannot always give an identical answer; it rests on the clinician to decide which test is most appropriate for the patient.

\section{Value of the cost/effectiveness analysis}

The evaluation of the cost of ELISA TB tests made in the publication [14] used by WHO to determine its policy is based on an analysis of a hypothetical Indian population. It states: "Since Anda-TB is likely to outperform more poorly studied in-house serological tests and less accurate rapid test formats, and laboratory accuracy is likely to exceed that in the field, our analysis likely overestimates the accuracy of serology". The 3 assumptions "likely" included in this single sentence applied to a virtual population invalidate the conclusions of the cost analysis. Contrary to the dubious claim made in this statement that rests on three unverified assumptions, the accuracy of the serology is by no means overestimated. Besides, the evaluation of a benefit versus cost is in itself subject to debate, as is the price of the serological test reported in the study. The correlation of cost with effectiveness [36] states that a year of life in the US is worth $\$ 50,000$ in medical bills. But is such a callous evaluation appropriate? What will be the opinion of a TB patient if the clinician proposes him to save a few rupees and leave him with the wrong diagnosis, the wrong prognosis, a treatment delayed by about 12.5 days to obtain confirmation by culture, or an untreated latent infection? In any case, our inquiries in the matter of price suggest that the real cost of a serological test for TB in India is $1 / 3$ that reported in the study: a survey of the prices in Delhi and Mumbai indicated a cost varying from 7 to $12 \$$. You will note that the price indicated in the cost-effectiveness study is surprisingly not substantiated by reference to any financial study or data.

\section{The Anda-TB Elisa test}

A60 antibodies in normal populations, non-tuberculosis patients and groups at risk: It has been shown that $4 \%$ of the general population in low TB incidence countries has low but significant levels of anti-A60 antibodies [37]. Additional research indicated that these $4 \%$ were restricted to two groups of persons, HIV-seropositive groups and drug addicts, both of which are high exposure groups [38]. It has been demonstrated that, within a group of workers, healthy persons in contact with the general population and therefore at higher risk of exposure, had higher anti-TB titres than those employed in administrative tasks [39]. Transplant patients and some cancer patients are also at risk $[7,40,41]$.

Production of antibodies in tuberculosis patients: The production of antibodies of classes IgG, IgM and IgA in pulmonary tuberculosis, in extra-pulmonary tuberculosis, in vaccinated infants has been abundantly analysed and demonstrated that these are variable due to spontaneous immune-depression induced by the pathogen, due to man-made depression due to the drugs used, and give valuable information that may be exploited to improve the treatment.

A patient devoid of IgG antibodies at entry may fail to produce significant amounts of IgG antibody during treatment but the treatment may eliminate the pathogen (negative cultures) without restoring his immunological defences. Low levels of antibodies at the start of the treatment may be timidly amplified during treatment. A high level of antibodies at entry may be amplified during treatment. Most interestingly, patients with low levels of antibody at entry, whose first three months of treatment do not improve antibodies production, may show a spectacular surge of antibodies and recovery when the clinician modifies the regimen [8].

Patients followed for IgG, IgM and IgA antibodies' production may produce none at the beginning of the first treatment but may increase them significantly as soon as treatment is started, and decline again to base level with recovery, which is disquieting. No sign of immunological booster effect can be observed on either IgG or IgA antibody production during a secondary infection, but well an unexpected production of IgM antibodies, as if the patient were without immunological memory.

TB antibodies' production in patients is personalised, sometimes associated with an immuno-depression affecting in a different ways all three classes of antibodies, and its value cannot be assessed with a statistical approach build on linear decreases.

\section{Conclusion}

Reduction of the TB diagnostic problem to antigen detection largely contributes to the current failure to eradicate this disease. Heterodox views should be evaluated on their merits and not excluded on sentimental grounds: bad news is not necessary false. If publications on humoral responses are given credit, evaluated properly and integrated [42-46], the following conclusions are drawn:

1. The humoral immune response to TB is just as important as the cellular one, and is subject to partial to total suppression by the pathogen. The depression may independently influence production of IgG, IgM, or IgA anti-TB antibodies in different patients. A heavy bacterial load may correspond to a negative serology.

2. One third of humanity is infected by a mycobacterial entity but most mycobacterial infections are latent, indicating that $\mathrm{TB}$ is an inefficient pathogen needing particular conditions to develop disease. Antibody detection is a good means to discover the latent cases prone to convert.

3. Mycobacterial infections revealed by the presence of antibodies are observed with variable frequency in non-tuberculosis patients suffering from diverse diseases. Not all diseases allow a mycobacterialsuperposed infection.

4. An immunosuppressive drug may demonstrate an anti-TB activity in vitro but its immunosuppressive activity will remain 
concealed. In patients, the immunosuppression of the drug will add to the immunosuppression induced by TB.

5. BCG rarely elicits IgG antibodies against the mycobacterial dominant A60-antigen in vaccinated newborns. Transferred maternal antibodies may impair the efficacy of the vaccination.

Contrary to the conclusions of the meta-analysis and of the costeffectiveness studies used by the WHO to determine its policy regarding TB and serodiagnosis, serological tests, at least those based on A60, have their use and are not as expensive as claimed.

The WHO policy statement rests on incorrect and invalid evidence. The experts who wrote the article used by the WHO to formulate its policy analysed a pool of published studies of widely divergent quality and drew thereupon conclusions not on the quality of the studies analysed but on the quality of the serological test itself. The metaanalysis is also fraught with errors. The first duty of meta-analysts, reviewers and policy makers is to evaluate the worth of the analysed and reviewed material and discard those publications that do not meet academic standards or are obviously marred with errors in design and technique, the second is to evaluate the validity of the conclusions drawn from correctly performed studies, and the third is to make correct recommendations based on these correct conclusions. The meta-analysis failed in this (and resulted in the WHO adopting a policy banning all serological tests for TB diagnosis).

The Western management of the TB problem has chosen to ignore the reality of the global situation and the urgency of solutions sensibly adapted to its needs. An efficient and responsible health system will evaluate basic new discoveries in clinical trials that lead to standards of good medical practice. These will themselves uncover the exceptions that will generate new basic investigations, new diagnostics and new therapeutics. Contrary to other fields of infectious diseases, contributions to $\mathrm{TB}$ made by investigators not belonging to the main stream have been rejected or ignored during decades, with emphasis maintained on antigen detection, standard-operating-procedures and directly-observed-therapy-short term. Unfortunately, this procedure has been shown to be imperfect. In view of this, it is laudable that the WHO and other groups such as FIND aim to reach a perfect diagnostic and therapeutic solution, but in view of the TB pathology, such aims may prove unattainable. On the other hand, at least in the field of diagnosis, we have tools that complement the standard ones and that are even more efficient than them in some groups of people. Let us determine their strengths and weaknesses and use them when required. The Indian health system evaluated the serology of TB in this spirit. Its use not only allowed to detect a large number of TB patients negative with the conventional methods, but it also allowed to uncover hitherto unsuspected aspects of the tuberculosis problem.

\section{Acknowledgments}

I sincerely thank Dr. HH Maes for his help, remarks and suggestions in the writing of this work.

\section{References}

1. Reggiardo Z, Middlebrook G (1974) Failure of passive serum transfer of immunity against aerogenic tuberculosis in rabbits. Proc Soc Exp Biol Med 145: 173-175.

2. Rennou M, Maes MC, Maes HH, Maes RF, Kidwai ZM, et al. (2016) A Antibodies against BCG and M. tuberculosis H37Ra do not Consistently Recognize Pathogenic M. tuberculosis whole Cells but Recognize their
Cytoplasmic Constituents. Implications for the Variability and Protective Efficacy of the Vaccine. Clin Microbiol 5: 2.

3. Chatterjee BR (1990) Controlled clinical trials. Comments on the contribution of Prof. Grosset. Int J Lepr 58: 376-8.

4. Glatman-Freedman A, Casadevall A (1998) Serum therapy for tuberculosis revisited: reappraisal of the role of antibody-mediated immunity against Mycobacterium tuberculosis. Clin Microbiol Rev 11: 514-532.

5. Enarson DA, Grosset J, Mwinga A, Hershfield ES, O'Brien R, et al. (1995) The challenge of tuberculosis: statements on global control and prevention. Lancet 346: 809-819.

6. Cocito CG (1991) Properties of the mycobacterial antigen complex A60 and its applications to the diagnosis and prognosis of tuberculosis. Chest 100: 1687-1693.

7. Kaustová J (1996) Serological IgG, IgM and IgA diagnosis and prognosis of mycobacterial diseases in routine practice. Eur J Med Res 1: 393-403.

8. Fadda G, Grillo R, Ginesu F, Santoru L, Zanetti S, et al. (1992) Serodiagnosis and follow up of patients with pulmonary tuberculosis by enzyme-linked immunosorbent assay. Eur J Epidemiol 8: 81-87.

9. Maes R, Homasson JP, Kubin M, Bayer M (1989) Development of an enzyme immunoassay for the serodiagnostic of tuberculosis and mycobacterioses. Med Microbiol Immunol 178: 323-335.

10. WHO TDR (2010) Commercial serodiagnostic tests for diagnosis of tuberculosis expert group meeting report.

11. WHO (2011) Commercial serodiagnostic tests for diagnosis of tuberculosis. Policy Statement.

12. Steingart KR, Flores LL, Dendukuri N, Schiller I, Laal S, et al. (2011) Commercial Serological Tests for the Diagnosis of Active Pulmonary and Extrapulmonary Tuberculosis: An Updated Systematic Review and MetaAnalysis. PLoS Med 8: e1001062.

13. American Statistical Association (2016) misuse of statistics for policy decisions. Nature 531: 151

14. Dowdy DW, Steingart KR, Pai M (2011) Serological testing versus other strategies for diagnosis of active tuberculosis in India: a cost-effectiveness analysis. PLoS Med 8: e1001074.

15. Guyatt GH, Oxman AD, Vist GE, Kunz R, Falck-Ytter Y, et al. (2008) GRADE: an emerging consensus on rating quality of evidence and strength of recommendations. BMJ 336: 924-926.

16. Lee DH, Vielemeyer O (2011) Analysis of Overall Level of Evidence Behind Infectious Diseases Society of America Practice Guidelines. Arch Intern Med 17: 18-22.

17. Stuebe AM (2011) Level IV evidence--adverse anecdote and clinical practice. N Engl J Med 365: 8-9.

18. Foulds J, O'Brien R (1997) New tools for the diagnosis of Tuberculosis: the perspective of low income countries. WHO Tuberculosis Diagnostic Workshop.

19. Gevaudan MJ, Bollet C, Charpin D, Mallet MN, De Micco P, et al. (1992) Serological response of tuberculosis patients to antigen 60 of BCG. Eur J Epidemiol 8: 666-676.

20. Alifano M, Del Pezzo M, Lamberti C, Faraone S, Covelli I, et al. (1994) ELISA method for evaluation of anti-A60 IgG in patients with pulmonary and extrapulmonary tuberculosis. New Microbiol 17: 37-43.

21. Alifano M, De Pascalis R, Sofia M, Faraone S, Del Pezzo M, et al. (1998) Detection of IgG and IgA against the mycobacterial antigen A60 in patients with extrapulmonary tuberculosis. Thorax 53: 377-380.

22. Delacourt C, Gobin J, Gaillard JL, de Blic J, Veron M, et al. (1993) Value of ELISA using antigen 60 for the diagnosis of tuberculosis in children. Chest 104: 393-398.

23. Rota S, Beyazova U, Karsligil T, CevheroÄŸlu C (1994) Humoral immune response against antigen 60 in BCG-vaccinated infants. Eur J Epidemiol 10: 713-718.

24. Turneer M, Van Nerom E, Nyabenda J, Waelbroeck A, Duvivier A, et al. (1994) Determination of humoral immunoglobulins $M$ and $G$ directed against mycobacterial antigen 60 failed to diagnose primary tuberculosis 
Citation: Maes R (2016) A Critical Appraisal of the Ban on Serological Tests for Tuberculosis Usefulness of Serological Monitoring of Tuberculosis Antibodies during the Treatment of TB Patients. Clin Microbiol 5: 254. doi:10.4172/2327-5073.1000254

Page 5 of 5

and mycobacterial adenitis in children. Am J Respir Crit Care Med 150: 1508-1512.

25. Singh P, Baveja CP, Talukdar B, Kumar S, Mathur MD, et al. (1999) Diagnostic Utility of ELISA Test Using Antigen A60 in Suspected Cases of Tuberculous Meningitis in Paediatric Age Group. Indian J Pathol Microbiol 42: 11-4.

26. Swaminathan S, Umadevi P, Shantha S, Radhakrishnan A, Datta M, et al (1999) Sero diagnosis of tuberculosis in children using two ELISA kits. Indian J Pediatr 66: 837-842.

27. Khalilzadeh S, Yazdanpanah S (2011) Sero diagnosis of tuberculosis in children using A60 antigens. Int J Tubercul Lung Dis 5: 200.

28. Gupta S, Bhatia R, Datta KK (1997) Serological diagnosis of childhood tuberculosis by estimation of mycobacterial antigen 60-specific immunoglobulins in the serum. Tuber Lung Dis 78: 21-27.

29. van der Werf TS, Das PK, van Soolingen D, Yong S, van der Mark TW, et al. (1992) Sero-diagnosis of tuberculosis with A60 antigen enzyme-linked immunosorbent assay: failure in HIV-infected individuals in Ghana. Med Microbiol Immunol 181: 71-76.

30. Pouthier F, Perriëns JH, Mukadi Y, Kayembe T, St Louis ME, et al. (1994) Anti-A60 immunoglobulin G in the serodiagnosis of tuberculosis in HIVseropositive and seronegative patients. AIDS 8: 1277-1280.

31. Del Pezzo M, De Filippis A, D'Alessio A, Rosiello M, Tosone G, et al (1990) A serological study of mycobacterial infections in AIDS and HIV-1 positive patients. G Batteriol Virol Immunol 83: 3-9.

32. Anderson BL, Welch RJ, Litwin CM (2008) Assessment of three commercially available serologic assays for detection of antibodies to Mycobacterium tuberculosis and identification of active tuberculosis. Clin Vaccine Immunol 15: 1644-1649.

33. Targeted tuberculin testing and treatment of latent tuberculosis infection (2000) This official statement of the American Thoracic Society was adopted by the ATS Board of Directors, July 1999. This is a Joint Statement of the American Thoracic Society (ATS) and the Centers for Disease Control and Prevention (CDC). This statement was endorsed by the Council of the Infectious Diseases Society of America. (IDSA), September 1999, and the sections of this statement. Am J Respir Crit Care Med 161: S221-247.
34. Sutherland I (1968) The ten-year incidence of clinical tuberculosis following "conversion" in 2550 individuals aged 14 to 19 years. TSRU Progress Report ; KNCV, The Hague, Netherlands.

35. Horsburgh CR (2004) Priorities for the treatment of latent tuberculosis infection in the United States. N Engl J Med 350: 2060-2067.

36. Wapner J (2011) Taking a Stab at Cost-Effectiveness PloS blog.

37. Mandler F (1991) Valutazione del valore predittivo del test sierologico in elisa A60 (TB-test) nelle diagnosi clinica di affezioni tubercolari. The Eurospital J Grado 5-14.

38. Maes R (1989) Incidence of inapparent active mycobacterial infections in France detected by an IgG serological test based on antigen 60 . Med Microbiol Immunol 178: 315-321.

39. Wirrmann C (1990) Public health application of a serological test for tuberculosis: study of the incidence of inapparent infections among the employees of an Alsatian supermarket. Eur J Epidemiol 6: 304-308.

40. Graham JC, Tweddle DA, Jenkins DR, Pollitt C, Pedler SJ, et al. (1998) Non-tuberculous mycobacterial infection in children with cancer. Eur J Clin Microbiol Infect Dis 17: 394-397.

41. Patel R, Roberts GD, Keating MR, Paya CV (1994) Infections due to nontuberculous mycobacteria in kidney, heart, and liver transplant recipients. Clin Infect Dis 19: 263-273.

42. Maes HH, Causse JE, Maes RF (1996) Mycobacterial infections: are the observed enigmas and paradoxes explained by immunosuppression and immunodeficiency? Med Hypotheses 46: 163-171.

43. Maes HH, Causse JE, Maes RF (1999) Tuberculosis I: a conceptual frame for the immunopathology of the disease. Med Hypotheses 52: 583-593.

44. Maes R (1991) Clinical usefulness of serological measurements obtained by antigen A60 in mycobacterial infections: development of a new concept. Klin Wochenschr 69: 696-709.

45. Maes RF (1999) Tuberculosis II: the failure of the BCG vaccine. Med Hypotheses 53: 32-39.

46. Maes RF (1999) The tuberculosis enigma: need for a new paradigm: importance of a knowledge of the immune status of the patients. Biomedicine 19: 1-14. 